\title{
A Case Report of Primary Resistance to EGFR TKI in Lung Adenocarcinoma Due to Coexisting MET Exon 14 Skipping Mutation with Excellent Response to Combination of Gefitinib and Capmatinib
}

\author{
1Department of Oncology, Bhaktivedanta Hospital and Research \\ Institute, Thane (East), Maharashtra, India \\ 2Department of Molecular Pathology, Neuberg Supratech, \\ Ahmedabad, Gujarat, India \\ ${ }^{3}$ Department of Radiology, Bhaktivedanta Hospital and Research \\ Institute, Thane (East), Maharashtra, India \\ ${ }^{4}$ Department of Medical Research Department, Bhaktivedanta \\ Hospital and Research Institute, Thane (East), Maharashtra, India
}

Nirmal Vivek Raut ${ }^{1}$ Siddharth Srivastava ${ }^{2}$ Guarav Dilip Gangwani ${ }^{3}$ Heena Sajid $\mathrm{Ali}^{4}$

\author{
Address for correspondence Nirmal Vivek Raut, Shrishti \\ Complex, Bhaktivedanta Swami Marg, Mira Road (East), Thane, \\ Maharashtra 401107, India (e-mail: drnirmalraut@gmail.com).
}

Ind J Med Paediatr Oncol 2021;42:204-207.

\begin{abstract}
Treatment of nonsmall cell lung cancer (NSCLC) carrying an epidermal growth factor receptor (EGFR) mutation depends on EGFR tyrosine kinase inhibitors (TKIs). However, all patients treated with EGFR TKI eventually develop progressive disease. Approximately, $20 \%$ of patients do not respond to EGFR TKIs, which is defined as primary resistance. The prognosis of these patients is similar to NSCLC with nondriver mutations. We report a case of a patient with EGFR exon 21 mutation who rapidly progressed in 15 days on Gefitinib. Next-generation sequencing (NGS) showed a MET exon

Keywords

- NSCLC

- Met Exon 14 Skip

- NGS

- Capmatinib 14 skip mutation coexisting with EGFR exon 21 mutation, causing primary resistance to EGFR TKI. Based on NGS reports, a treatment combining Gefitinib and Capmatinib, a MET inhibitor, induced a rapid response in the patient, which was sustained at the end of 8 months. This clearly emphasizes the need for comprehensive genomic profiling using NGS over single gene testing.
\end{abstract}

\section{Introduction}

Lung cancer is the poster child of precision oncology, which views cancer through the lens of single oncogenic alteration and is also known as driver mutation. Current clinical research is focused on improving outcomes by targeting its mutant protein, namely, epidermal growth factor receptor (EGFR), ALK, ROS1, BRAF, Her2, etc. In this existing paradigm, mutations other the driver mutation are considered as passengers with little to no impact on clinical outcomes. We present a case with coexisting EGFR L858R and MET Exon
14 skipping mutation and not responding to single agent EGFR tyrosine kinase inhibitor (TKI).

Hence, we advocate next-generation sequencing (NGS) panel testing at outset.

\section{Case History}

A 44-year-old, nonsmoker woman presented with a 3-month history of chest pain, cough, and dyspnea. Her Eastern Cooperative Oncology Group performance status (ECOG PS) was 1 . CT scan showed $8.8 \times 8.3 \times 9.4 \mathrm{~cm}$ mass in the
DOI https://doi.org/ $10.1055 / \mathrm{s}-0041-1731851$ ISSN 0971-5851
(C) 2021. Indian Society of Medical and Paediatric Oncology.

This is an open access article published by Thieme under the terms of the Creative Commons Attribution-NonDerivative-NonCommercial-License, permitting copying and reproduction so long as the original work is given appropriate credit. Contents may not be used for commercial purposes, or adapted, remixed, transformed or built upon. (https://creativecommons.org/licenses/by-nc-nd/4.0/).

Thieme Medical and Scientific Publishers Private Ltd. A-12, Second Floor, Sector -2, NOIDA -201301, India 


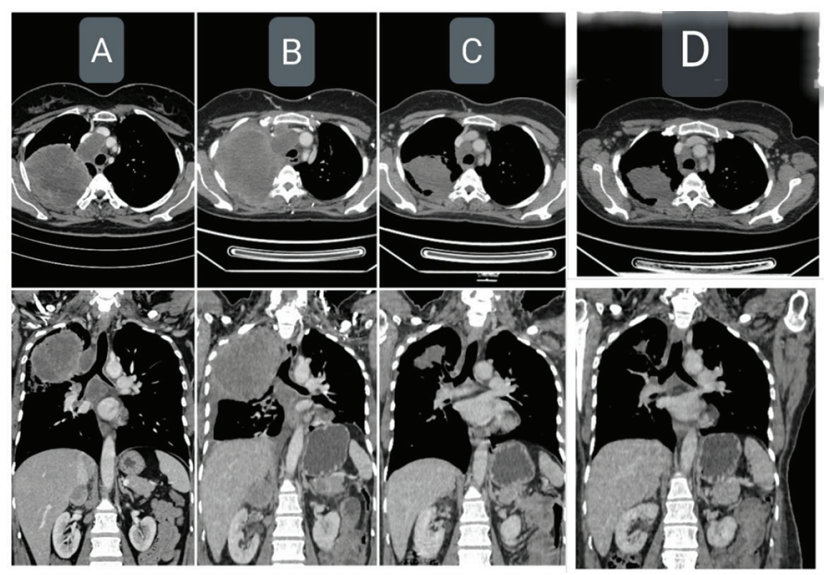

Fig. 1 Contrast chest CT axial images (upper panels) and coronal images (lower panels) during initial diagnosis (A); follow-up visit when the patient was unresponsive to Gefitinib (B); and next follow-up 4.5 months after Capmatinib along with Gefitinib (C). Persistent response seen at the end of on combination of Gefitinib and Capmatinib (D).
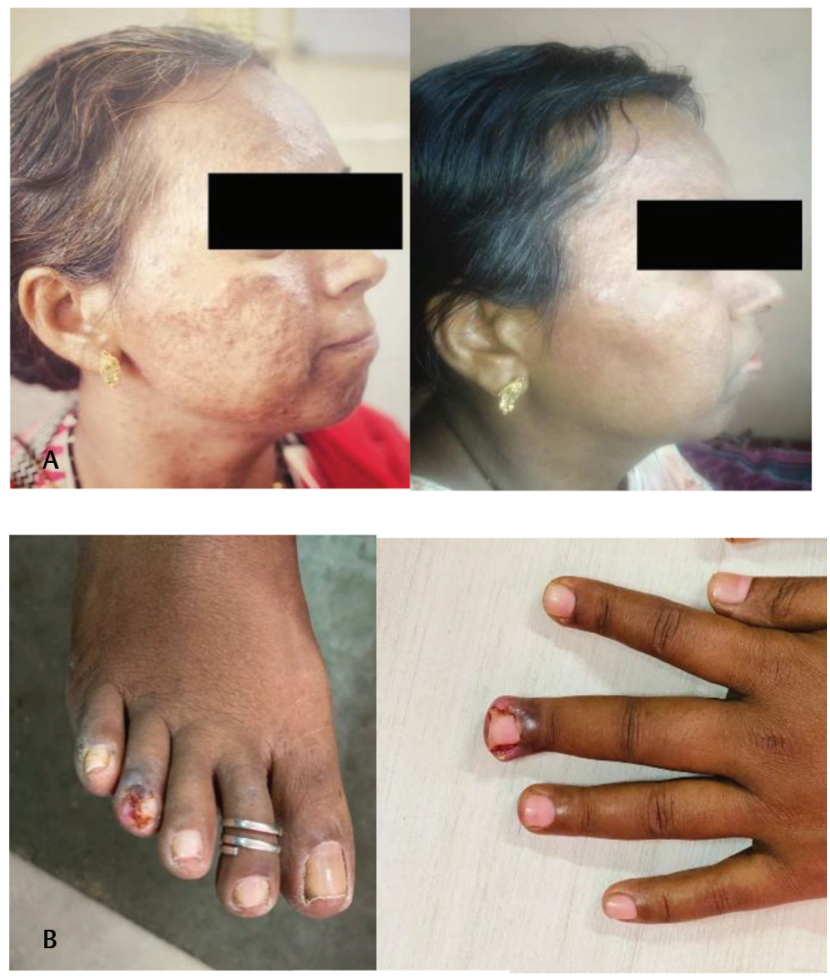

Fig. 2 (A)Adverse effect-acneiform rash (common terminology criteria for adverse events [CTCAE] v5.0). Left-grade 3 within 15 days of starting Gefitinib. Right-grade 2 after 8 months of Gefitinib plus Capmatinib. (B)Adverse effect-paronychia grade 2 (CTCAE v5.0) at end of 8 months of Gefitinib plus Capmatinib combination.

right lung upper lobe. There was evidence of lymphangitis carcinomatosis in the right middle and lower lobe ( - Fig. 1 A). These findings were suggestive of stage IV disease as per the 8th edition of the American Joint Committee on Cancer. Histopathology established the diagnosis as TTF1 positive lung adenocarcinoma. Subsequent Sanger sequencing detected p.L858R pathogenic mutation in exon 21 of the
EGFR gene. Patient was started on Gefitinib $250 \mathrm{mg} /$ day, within 15 days of which the patient developed progressive dyspnea and new onset cervical lymphadenopathy. Positron emission tomography (PET) scan confirmed disease progression (-Fig. 1 B). Her ECOG PS now ("at progression") was 4 and she required admission in hospital. She also developed grade 2 acneiform rash as per common terminology criteria for adverse events (CTCAE) version 5.0 and grade 2 paronychia ( - Fig. 2 A, B).

\section{A}

NP_005219

NG_007726
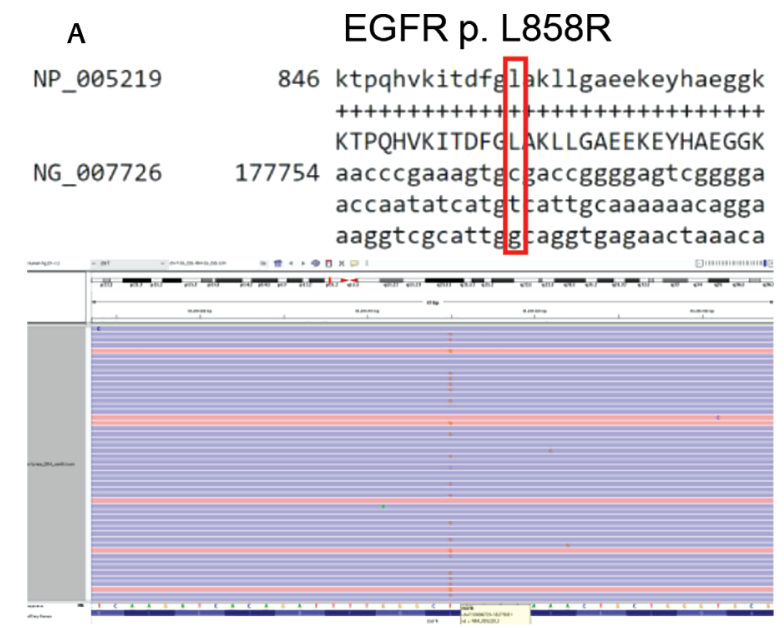

B

\section{MET Exon 14 splice}
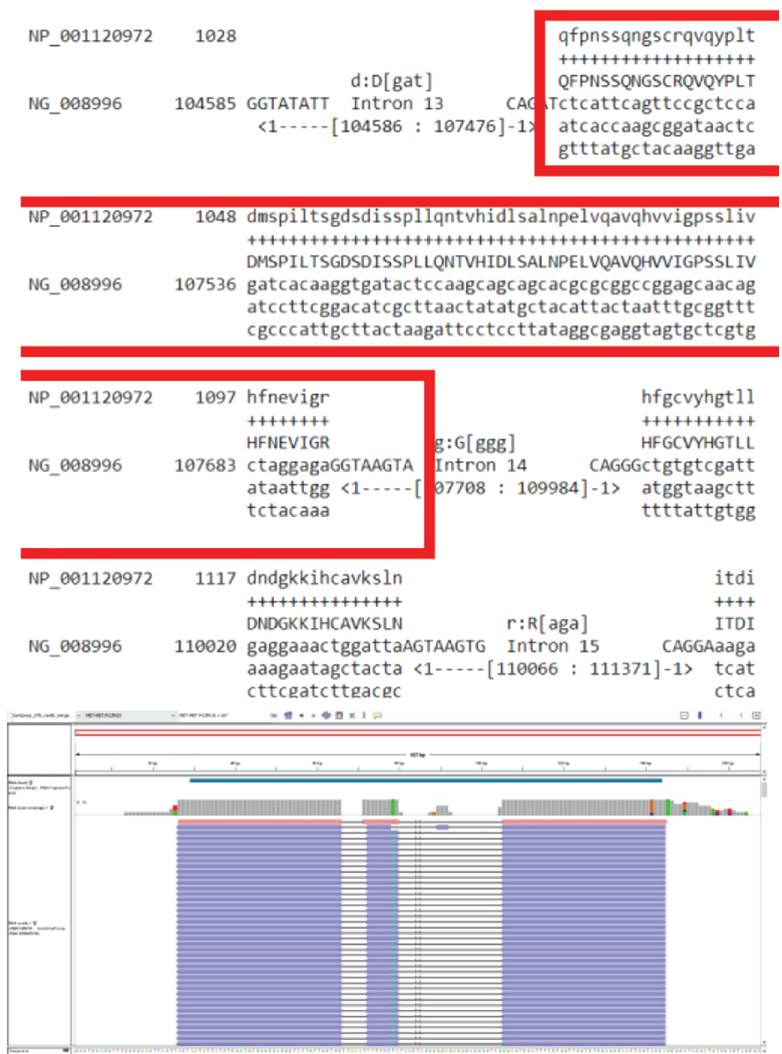

Fig. 3 (A, B) Next-generation sequencing (NGS) report showing epidermal growth factor receptor (EGFR) p.L858R and MET exon 14 splice mutations. Total mapped fusion panel reads -329059 . Read counts of MET Exon skipping $=833$. 
Table 1 Radiographic response

\begin{tabular}{|c|c|c|c|c|c|c|}
\hline Time points & & $15 / 8 / 19$ & $11 / 10 / 19$ & $28 / 11 / 19$ & $22 / 2 / 20$ & $9 / 6 / 20$ \\
\hline Treatment & & Pretreatment & $\begin{array}{l}15 \text { days } \\
\text { Gefitinib }\end{array}$ & $\begin{array}{l}6 \text { weeks Gef + } \\
\text { Capmatinib }\end{array}$ & $\begin{array}{l}4.5 \text { months } \\
\text { Gef + Cap }\end{array}$ & $\begin{array}{l}8 \text { months } \\
\text { Gef + Cap }\end{array}$ \\
\hline \multirow[t]{2}{*}{ Right lung mass } & Size & $8.8 \times 8.3^{a}$ & $10.5 \times 10$ & $6.5 \times 6.0$ & $5.4 \times 6$ & $5.4 \times 5.5$ \\
\hline & SUV & $N / A^{b}$ & 18.52 & 4.40 & 2.13 & 1.58 \\
\hline \multirow[t]{2}{*}{ Right para-tracheal LN } & Size & $4.3 \times 2.8$ & $3.5 \times 2.5$ & $2.2 \times 1.5$ & $1.9 \times 2.1$ & $1.5 \times 2.1$ \\
\hline & SUV & $N / A^{b}$ & 9.61 & 4.13 & 3.07 & 2.92 \\
\hline \multirow[t]{2}{*}{ Right adrenal } & Size & $2.6 \times 3.0$ & $5.8 \times 5.2$ & CR & CR & CR \\
\hline & SUV & $\mathrm{b}$ & 22.58 & & & \\
\hline Pleural effusion & & None & Moderate & Minimal & None & None \\
\hline
\end{tabular}

Abbreviations: CR, complete response; SUV, specific uptake value; LN, lymph node; N/A, not applicable, Gef, Gefitinib; Cap, Capmatinib

${ }^{a}$ All sizes are in $\mathrm{cm}$.

b Pretreatment scan on August 15, 2019, was a multidetector CT scan with no metabolic scan done at that time. Hence, SUV was unknown before therapy initiation.

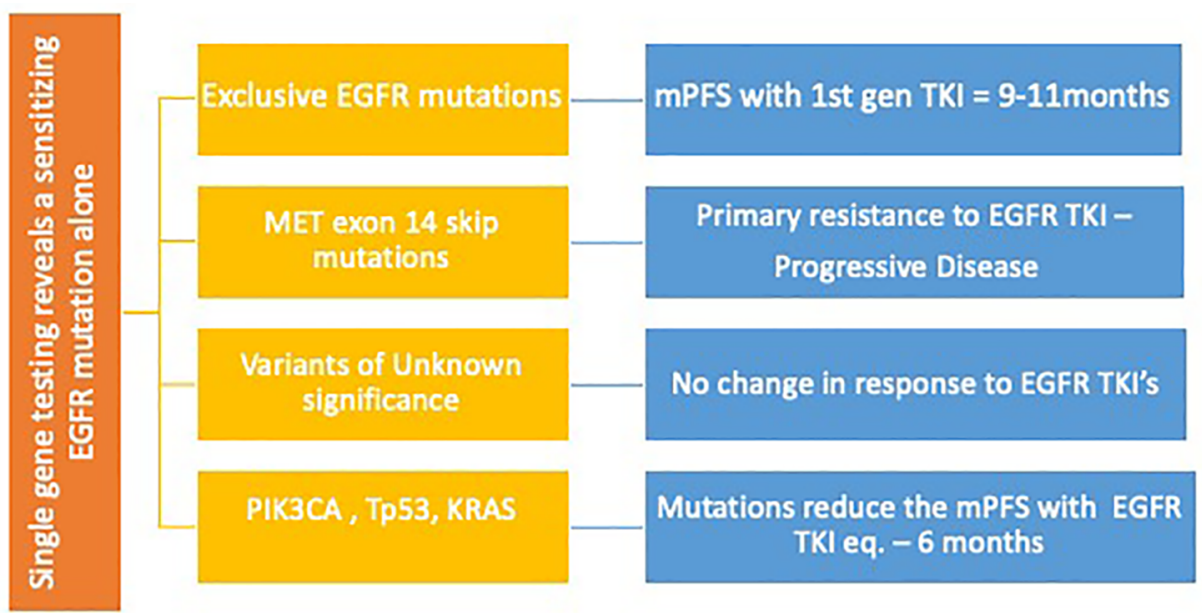

\section{$\begin{array}{lll}\text { RT-PCR / } & \text { NGS reveals concurrent } & \text { Clinical Implications } \\ \text { Sanger } & \text { mutations with EGFR }\end{array}$}

Fig. 4 New proposed pragmatic classification for nonsmall cell lung cancer (NSCLC) with epidermal growth factor receptor (EGFR) mutations.

As a part of the institutional protocol, multigene testing by NGS was performed on tissue for initial diagnosis. The test includes single nucleotide variations in EGFR, ALK, KRAS, BRAF, MET, RET and ERBB2, copy number variants of MET, and gene fusions of ALK and ROS1. NGS reports of this patient detected MET Exon 14 skipping along with EGFR p. L858R ( - Fig. 3 A, B). Based on this, we added Capmatinib, a type Ib MET inhibitor. The patient was enrolled in a managed access program by Novartis. Capmatinib was given $400 \mathrm{mg}$ twice daily along with Gefitinib (250 mg). This dose was determined on the basis of RP2D from a phase Ib/II trial. ${ }^{1}$ After 14 days of therapy, dyspnea and cervical lymph nodes resolved. Acneiform rash, which developed on Gefitinib, remained stable despite the introduction of Capmatinib. An early response evaluation PET scan at 6 weeks showed partial response (PR) with significant reduction in metabolic activity of the previously noted right lung mass. At the end of 4.5 months, with same dose of Gefitinib and Capmatinib, the patient continued to respond ( - Fig. 1 C). She has no adverse effects except grade 2 skin rash and grade 1 paronychia (-Fig. 2 A, B)
The follow-up scan (-Fig. 1 D) at the end of 8 months showed only a right lung mass measuring $5.5 \times 5.0 \mathrm{~cm}$ and mediastinal nodes reduced further in size, suggestive of continued combination therapy at full doses ( $\mathbf{- T a b l e ~} \mathbf{1}$ ).

\section{Discussion}

MET Exon 14 skip mutations are present in 3 to $4 \%$ of nonsmall cell lung cancer (NSCLC) with higher frequency in elderly, smokers, and sarcomatoid histology. ${ }^{2}$ Of all MET alterations, Exon 14 skip mutations are the best predictive biomarkers of response to MET inhibitors, and a RNA-based NGS is preferable to capture these mutations. These mutations are mutually exclusive with other driver mutations, although coexisting MET amplification is reported.,23 Barring some anecdotal cases, this is the first case of lung adenocarcinoma with de novo co-existing EGFR L858R and MET Exon 14 skip mutation successfully treated with a combination of Gefitinib and Capmatinib. ${ }^{4,5}$ The duration of response to targeted therapy is affected by development of acquired 
resistance, which is the Achilles heel of precision oncology. In our case, there was significant reduction in the size and metabolic activity as early as 6 weeks, and this response is still sustained until 8 months. In similar cases reported earlier, adverse effects to combination of EGFR TKI and MET inhibitors warranted dose adjustments. In this case, the patient is still on full dose of Gefitinib and Capmatinib with no added toxicities. Limitations in this case was not reconfirming MET alteration by reverse transcription polymerase chain reaction (RT-PCR) and administering first-generation TKI Gefitinib instead of third-generation TKI-Osimertinib, which is the current standard of care in first-line treatment. ${ }^{6}$ This can be explained by the lower gross domestic product (GDP) of our country, with most patients paying out of pocket. However, it is unlikely that that this patient would have responded if given Osimertinib instead of Gefitinib. Studies exploring acquired resistance mechanisms to first-line Osimertinib show incidence of MET alterations which is, in fact, higher than Gefitinib.

Lung cancer research has been successful in prolonging median progression-free survival (PFS) from 9 months to 18 months. ${ }^{6}$ However, the overall response rates (ORR) with Gefitinib (76\%) is not very different from Osimertinib arm (80\%). Today, NGS is utilized more on progression after the first-line TKI, because of the heterogenous mechanisms of resistance and concurrent mutations. But our case emphasizes the need of NGS even at baseline. In the FLAURA study, patients' tissue biopsies were done at baseline. Doing liquid biopsies at baseline will uncover more coexisting mutations along with EGFR, as it allows more comprehensive analysis of tumor heterogeneity. A cfDNA analysis of 1122 EGFR mutant NSCLC showed the incidence of concurrent mutations was as high as $92 \%{ }^{7}$ This study defined new pathways of limiting EGFR TKI response, including Wnt/Beta catenin and CDK 4/6 cell cycle genes. Another multivariate analysis by Kim et al showed that mutations in TP53, RB1, PTEN and MDM2 were poor prognostic factors in patients on Osimertinib. ${ }^{8}$ Categorizing cases as one described in this case report as TKI refractory and treating them with conventional chemotherapy will take us back 15 to 20 years in terms of prognosis.

NSCLC with EGFR mutations is a heterogenous subgroup, with varying responses to TKI, based on coexisting mutations. We would like to propose a new classification, as depicted in - Fig. 4.

\section{Conclusion}

This case emphasizes the importance of NGS over single gene testing as it can find concurrent mutations which can strongly influence the response of targeted therapy to the primary driver mutation leading to improved treatment outcomes.

\section{Funding}

This research received no specific grant from any funding agency in the public, commercial, or not-for-profit sectors.

\section{Conflict of Interest}

None of the authors of this paper have a financial or personal relationship with other people or organizations which could inappropriately influence or bias the content of the paper.

\section{References}

1 Wu YL, Zhang L, Kim DW, et al. Phase Ib/II study of Capmatinib (INC280) plus Gefitinib after failure of epidermal growth factor receptor (EGFR) inhibitor therapy in patients with EGFR-mutated, MET factor-dysregulated non-small-cell lung cancer. J Clin Oncol 2018;36(31):3101-3109

2 Schrock AB, Frampton GM, Suh J, et al. Characterization of 298 patients with lung cancer harboring MET Exon 14 skipping alterations. J Thorac Oncol 2016;11(9):1493-1502

3 Awad MM, Oxnard GR, Jackman DM, et al. MET Exon 14 mutations in non-small-cell lung cancer are associated with advanced age and stage-dependent MET genomic amplification and c-Met overexpression. J Clin Oncol 2016;34(7):721-730

4 Li W, Kang J, Zhang X, et al. Coexistence of MET exon 14 mutations with EGFR mutations in non-small cell lung cancer. J Clin Oncol 2017;35(15 suppl):e20636-e20636

5 Kauffmann-Guerrero D, Kahnert K, Kumbrink J, Syunyaeva Z, Tufman A, Huber RM. Successful treatment of a patient with NSCLC harboring an EGFR mutation and a concomitant Met Exon 14 skipping mutation combining Afatinib and Crizotinib. Clin Lung Cancer 2019;20(1):59-62

6 Soria JC, Ohe Y, Vansteenkiste J, et al. FLAURA Investigators. Osimertinib in untreated EGFR-mutated advanced non-small-cell lung cancer. N Engl J Med 2018;378(2):113-125

7 Blakely CM, Watkins TBK, Wu W, et al. Evolution and clinical impact of co-occurring genetic alterations in advanced-stage EGFR-mutant lung cancers. Nat Genet 2017;49(12):1693-1704

8 Kim Y, Lee B, Shim JH, et al. Concurrent genetic alterations predict the progression to target therapy in EGFR-mutated advanced NSCLC. J Thorac Oncol 2019;14(2):193-202 